\title{
Genomic islands from five strains of Burkholderia pseudomallei
} Apichai Tuanyok11, Benjamin R Leadem11, Raymond K Auerbach1, Stephen M Beckstrom-Sternberg1,2, James S Beckstrom-Sternberg1, Mark Mayo ${ }^{3}$, Vanaporn Wuthiekanun ${ }^{4}$, Thomas S Brettin ${ }^{5}$, William C Nierman ${ }^{6,7}$, Sharon J Peacock ${ }^{4}$, Bart J Currie ${ }^{3}$, David M Wagner ${ }^{1}$ and Paul Keim*1,2

\author{
Address: ${ }^{1}$ Department of Biological Sciences, Northern Arizona University, Flagstaff, AZ, 86011-5640, USA, ${ }^{2}$ The Translational Genomics Research \\ Institute, 445 N. Fifth St. Phoenix, AZ, 85004, USA, ${ }^{3}$ Menzies School of Health Research, Charles Darwin University, Northern Territory, Australia, \\ ${ }^{4}$ Mahidol-Oxford Research Unit, Mahidol University, Bangkok, Thailand, ${ }^{5}$ Los Alamos National Laboratory, Los Alamos, NM, 87545, USA, ${ }^{6}$. \\ Craig Venter Institute, 9704 Medical Center Drive, Rockville, MD 20850, USA and ${ }^{7}$ George Washington University, 2300 Eye Street, NW, \\ Washington DC, 20037, USA \\ Email: Apichai Tuanyok - Apichai.Tuanyok@nau.edu; Benjamin R Leadem - Benjamin.Leadem@nau.edu; \\ Raymond K Auerbach - Raymond.Auerbach@yale.edu; Stephen M Beckstrom-Sternberg - sbeckstrom@tgen.org; James S Beckstrom- \\ Sternberg - jamiebs45@yahoo.com; Mark Mayo - Mark.Mayo@menzie.edu.au; Vanaporn Wuthiekanun - Lek@tropmedres.ac; \\ Thomas S Brettin - brettin@lanl.gov; William C Nierman - wnierman@tigr.org; Sharon J Peacock - sharon@tropmedres.ac; \\ Bart J Currie - Bart.Currie@menzies.edu.au; David M Wagner - Dave.Wagner@nau.edu; Paul Keim* - Paul.Keim@nau.edu \\ * Corresponding author
}

Published: 27 November 2008

BMC Genomics 2008, 9:566 doi:10.1186/147|-2164-9-566

This article is available from: http://www.biomedcentral.com/I47I-2164/9/566

(c) 2008 Tuanyok et al; licensee BioMed Central Ltd.

This is an Open Access article distributed under the terms of the Creative Commons Attribution License (http://creativecommons.org/licenses/by/2.0), which permits unrestricted use, distribution, and reproduction in any medium, provided the original work is properly cited.

\begin{abstract}
Background: Burkholderia pseudomallei is the etiologic agent of melioidosis, a significant cause of morbidity and mortality where this infection is endemic. Genomic differences among strains of $B$. pseudomallei are predicted to be one of the major causes of the diverse clinical manifestations observed among patients with melioidosis. The purpose of this study was to examine the role of genomic islands (Gls) as sources of genomic diversity in this species.
\end{abstract}

Results: We found that genomic islands (Gls) vary greatly among $B$. pseudomallei strains. We identified $7 \mathrm{l}$ distinct $\mathrm{Gls}$ from the genome sequences of five reference strains of $B$. pseudomallei: K96243, I7I0b, II06a, MSHR668, and MSHR305. The genomic positions of these Gls are not random, as many of them are associated with tRNA gene loci. In particular, the 3' end sequences of tRNA genes are predicted to be involved in the integration of Gls. We propose the term "tRNAmediated site-specific recombination" (tRNA-SSR) for this mechanism. In addition, we provide a GI nomenclature that is based upon integration hotspots identified here or previously described.

Conclusion: Our data suggest that acquisition of Gls is one of the major sources of genomic diversity within $B$. pseudomallei and the molecular mechanisms that facilitate horizontally-acquired Gls are common across multiple strains of $B$. pseudomallei. The differential presence of the 7l Gls across multiple strains demonstrates the importance of these mobile elements for shaping the genetic composition of individual strains and populations within this bacterial species. 


\section{Background}

Burkholderia pseudomallei is the causative agent of melioidosis, an important tropical disease affecting people in Southeast Asia and the tropical "Top End" of northern Australia. Within these regions, this Gram-negative bacterium exists in the environment as a soil saprophyte and accounts for $20 \%$ of community-acquired septicemias and may cause death in $40 \%$ of treated patients [1]. Reinfection and relapse are common in melioidosis patients $[2,3]$. Infection can occur when contaminated soil or water comes into contact with breaks in the skin; this is a common infection route for rice farmers in northeastern Thailand $[4,5]$. Inhalation of contaminated dust has also been confirmed as a route of infection, including U.S. helicopter pilots during the Vietnam War [6]. Because of the high inhalational risk, the U.S. Centers for Disease Control and Prevention have classified B. pseudomallei as a Category B Select Agent [7]. Various clinical manifestations are associated with melioidosis, ranging from subclinical involvement to symptomatic characteristics that may include localized cutaneous infection, acute pulmonary infection, bacteremia, and disseminated infection $[1,8,9]$. Cheng and Currie [10] suggested that this variation in clinical presentation may be caused by one or more of three factors: variation in mode of acquisition, variation in host immune response, or variation among bacterial strains, including presence/absence of virulence factors.

Many challenges exist for the control and prevention of melioidosis [9]. At present, cellular and molecular mechanisms associated with these diverse clinical manifestations are not fully understood. Vaccine development and better therapeutics are necessary to prevent and treat melioidosis. However, knowledge of the relationship between hosts and pathogen is still limited. This has hindered improved vaccine and therapeutic developments, which require a full understanding of genomics and bacterial pathogenesis.

After the first genome of B. pseudomallei K96243 was released in 2004 [11], subsequent studies capitalized upon this foundation, which lead to enhanced genetic and genomic analyses that have facilitated a better understanding of this organism. As additional genomic sequences have been generated, striking differences have been observed. For example, two mutually exclusive gene cassettes, termed "BTFC and YLF", have been described that are dissimilar in their geographical distribution [12].

It is well established that B. pseudomallei contains an "open" genome [13] that recombines at a high frequency, leading to great intra-species diversity within and among pathogen populations. In recent unpublished analyses, we have found that when compared to existing genomic sequences, new B. pseudomallei genome sequences can contain as much as $500 \mathrm{~Kb}$ of additional genomic material in the form of blocks of novel DNA known as genomic islands. Thus, we hypothesize that the primary differences among $B$. pseudomallei genomes are horizontal gene transfer events from diverse bacterial or phage origins. Horizontal gene transfer involves the incorporation of genetic elements, perhaps directly into the genome where they form genomic islands [14]. Currently, very little is known about fitness in $B$. pseudomallei. It seems likely that the genes contained in genomic islands may generate unique phenotypes and affect bacterial fitness, such as the interaction of bacterial cells with their surrounding environment. Fitness phenotypes could range from the ability to survive under extreme environmental conditions to the ability to defeat host immune system defenses.

Here, we describe diversity among $B$. pseudomallei genomes in terms of identification and differential possession of genomic islands. Nomenclature for genomic islands is proposed, and the specific mechanism behind the genetic recombination is explained in detail. Finally, we present the frequency distributions of a focused group of interesting genomic islands across a large, diverse collection of $B$. pseudomallei isolates.

\section{Results and discussions \\ Identification of genomic islands in B. pseudomallei \\ i) Genomic comparison of five B. pseudomallei strains}

We identified genomic islands in B. pseudomallei from a set of 5 diverse reference genomes: strains K96243, 1710b, 1106a, MSHR668, and MSHR305 (Table 1). All 5 strains were isolated from melioidosis patients in Thailand or Australia. Clinical manifestations and severity of disease caused by these 5 strains were varied. Thai patients infected by strains K96243, 1710b and 1106a exhibited classical melioidosis manifestations, including lung and liver abscesses, and septicemia. In contrast, Australian patients infected by strains MSHR668 and MSHR305 had the relatively rare melioidosis encephalomyelitis and neurological involvement. Patients infected with strains K96243, 1710b, and MSHR305 died. Detailed clinical information associated with all five isolates, as well as their genomic features, is summarized in Table 1.

The five genomes were compared using Artemis Comparison Tool (ACT) [15], which enabled genomic alignment and visualization of BLASTN results. We confirmed that all genome sequences contained 2 chromosomes, except the genome sequence of MSHR305, which was incomplete at the time of writing this manuscript. To facilitate genomic comparison with the other 4 strains, we created 2 artificial chromosomes from the genome contigs of strain MSHR305 [see Additional file 1: Figure S1a and S1b 
Table I: Information about the five B. pseudomallei strains utilized in the genomic island analyses.

\begin{tabular}{|c|c|c|c|c|}
\hline \multirow[t]{2}{*}{ Strain } & \multirow[t]{2}{*}{ Clinical Information } & \multicolumn{2}{|c|}{ Genomic information } & \multirow[t]{2}{*}{ Source } \\
\hline & & Size & $\begin{array}{c}\text { Accession no. \& Genome } \\
\text { Center }\end{array}$ & \\
\hline K96243 & $\begin{array}{l}\text { Isolated in } 1996 \text { in Khon Kaen, Thailand } \\
\text { from a } 34 \text { year old female diabetic patient } \\
\text { with a clinical history of short incubation, } \\
\text { septicemic infection, and rapid progression } \\
\text { to death. }\end{array}$ & Chrl: $4.07 \mathrm{Mb}$; Chr2: $3.17 \mathrm{Mb}$ & $\begin{array}{l}\text { BX571965 and BX571966 Sanger } \\
\text { Institute }\end{array}$ & Dr. S. Songsivilai* \\
\hline $1710 b$ & $\begin{array}{l}\text { Isolated in } 1999 \text { in Ubon Ratchathani, } \\
\text { Thailand from blood collected from a } 55 \\
\text { year old male patient with known primary } \\
\text { melioidosis in 1996. This second episode } \\
\text { represented relapse with the same strain. } \\
\text { The patient died. }\end{array}$ & Chrl: $4.13 \mathrm{Mb}$; Chr2: $3.18 \mathrm{Mb}$ & CPO00I 24 and CP000I $25 \mathrm{JCVI}$ & Dr. S. Peacock \\
\hline $1106 a$ & $\begin{array}{l}\text { Isolated in } 1993 \text { in Ubon Ratchathani, } \\
\text { Thailand from pus aspirated from liver } \\
\text { abscess from a } 23 \text {-year old female rice } \\
\text { farmer. The patient survived. }\end{array}$ & Chrl: $3.99 \mathrm{Mb}$; Chr2: $3.10 \mathrm{Mb}$ & CP000572 and CP000573 JCVI & Dr. S. Peacock \\
\hline MSHR668 & $\begin{array}{l}\text { Isolated in } 1995 \text { in Darwin, Australia from } \\
\text { the blood of a } 53 \text { year old male patient } \\
\text { with severe melioidosis encephalomyelitis. } \\
\text { The patient survived. }\end{array}$ & Chrl: 3.9। Mb; Chr2: $3.13 \mathrm{Mb}$ & CP000570 and CP00057I JCVI & Dr. B. Currie \\
\hline MSHR305 & $\begin{array}{l}\text { Isolated in } 1994 \text { from an autopsy sample } \\
\text { from the brain of a fatal melioidosis } \\
\text { encephalomyelitis case at the Royal } \\
\text { Darwin Hospital, Northern Territory, } \\
\text { Australia. Neurological melioidosis is rare, } \\
\text { occurring only in approximately } 5 \% \text { of } \\
\text { diagnosed cases. }\end{array}$ & 36 contigs $7,453,647 \mathrm{nt}$ & AAYX00000000 JGI & Dr. B. Currie \\
\hline
\end{tabular}

*Described in [II].

JCVI (J. Craig Venter Institute); JGI (Joint Genome Institute)

for circular genomic structures], recognizing that this genomic arrangement is not confirmed.

In general, we identified genomic islands by identifying variable regions among the five genomes and determining if any of these regions met the general criteria previously described by Holden et al. in their analysis of strain K96243 [11], or the criteria described by Hacker and Kaper [16]. These criteria included: size of large inserts, distinct $\% \mathrm{G}+\mathrm{C}$ compared to the rest of the genome, and the presence of mobility genes. It is important to note that across the 5 strains multiple GIs were often present at the same genomic location (Table 2 ). In practice, our identification of genomic islands involved four main steps.

First, we determined if the 16 specific GIs previously identified in K96243 [11] were present in the four other strains. In K96243, 12 of these GIs are found on chromosome 1 and four are found on chromosome 2. Using the genomic locations of these GIs in K96243 as a reference, we performed pair-wise comparisons between K96243 and the other four strains and noted the differential presence of the 16 GIs in the other four strains. These 16 specific GIs were uncommon in the other $4 \mathrm{~B}$. pseudomallei genomes: only four of these GIs (GI1, GI6, GI10, and GI14) were found in at least one of the other four strains, and none were found in all of the other strains (Table 2).

Second, we identified new, distinct GIs that were present at the same 16 genomic locations where GIs were previously found in K96243. To accomplish this, we used ACT to align the genomes of all five strains in the following order: K96243 vs. $1710 \mathrm{~b}$ vs. $1106 \mathrm{a}$ vs. MSHR668 vs. MSHR305. In addition to identifying structural variation among any GIs present at these 16 locations, this alignment also allowed us to locate integration sites. Even when there is more than one GI found at the same relative genomic location, the integration sites were all similar across the five strains and these integrations sites were often associated with tRNA genes (Table 3 ). The genomic locations of GI2, GI5, and GI9 in K96243 did not contain GIs in any of the other four strains. One of the other locations, corresponding to GI6 in K96243, contained an identical GI in just strain MSHR305. However, the GIs located at the 13 other locations were quite diverse: at four of these locations each of the five strains possessed a distinct GI containing a different set of genes (Table 2). 
Table 2: 7 I GIs identified among five strains of B. pseudomallei.

\begin{tabular}{|c|c|c|c|c|c|c|c|c|}
\hline Strains & K96243* & $1710 b$ & $1106 a$ & MSHR668 & MSHR305 & Strains with Gls & Unique Gls & \% Unique \\
\hline \multirow[t]{25}{*}{ Chromosome I } & GII & GII & GII & GII.I & - & 4 & 2 & $50 \%$ \\
\hline & - & - & - & GIla & - & I & 1 & $100 \%$ \\
\hline & $\mathrm{Gl} 2$ & - & - & - & - & I & 1 & $100 \%$ \\
\hline & $\mathrm{GI3}$ & GI3.I & - & - & - & 2 & 2 & $100 \%$ \\
\hline & - & - & - & - & Gl3a & I & I & $100 \%$ \\
\hline & Gl4 & Gl4.I & Gl4.2 & Gl4.3 & Gl4.4 & 5 & 5 & $100 \%$ \\
\hline & GI5 & - & - & - & - & I & 1 & $100 \%$ \\
\hline & - & Gl5a & GI5a.I & GI5a.I & Gl5a.2 & 4 & 3 & $75 \%$ \\
\hline & G16 & - & - & - & G16 & 2 & I & $50 \%$ \\
\hline & - & - & Gl6a & - & - & I & 1 & $100 \%$ \\
\hline & - & Gl6b & - & - & - & I & I & $100 \%$ \\
\hline & Gl7 & GI7.I & GI7.2 & GI7.3 & GI7.4 & 5 & 5 & $100 \%$ \\
\hline & Gl8 & GI8.I & GI8.2 & - & Gl8.3 & 4 & 4 & $100 \%$ \\
\hline & - & - & - & Gl8a & - & I & 1 & $100 \%$ \\
\hline & - & GI8b & GI8b & - & - & 2 & 1 & $50 \%$ \\
\hline & - & - & - & - & GI8c & 1 & I & $100 \%$ \\
\hline & - & - & - & - & GI8d & I & I & $100 \%$ \\
\hline & G19 & - & - & - & - & 1 & I & $100 \%$ \\
\hline & - & - & - & Gl9a & - & I & I & $100 \%$ \\
\hline & - & - & - & GI9b & - & I & 1 & $100 \%$ \\
\hline & - & - & - & $\mathrm{G} 19 \mathrm{c}$ & GI9c.I & 2 & 2 & $100 \%$ \\
\hline & GIIO & GII0.I & GII0.2 & GIIO & GII0.3 & 5 & 4 & $80 \%$ \\
\hline & GIII & - & GIII.I & - & GIII.2 & 3 & 3 & $100 \%$ \\
\hline & GII2 & GII2.I & GII 2.2 & GII2.3 & GII 2.4 & 5 & 5 & $100 \%$ \\
\hline & - & - & GII $2 a$ & - & - & I & 1 & $100 \%$ \\
\hline \multirow[t]{12}{*}{ Chromosome 2} & GII3 & GII3.I & GII 3.2 & GII 3.3 & GII 3.4 & 5 & 5 & $100 \%$ \\
\hline & GII4 & GII4 & GII 4 & GII4.I & GII4 & 5 & 2 & $40 \%$ \\
\hline & - & - & - & - & $\mathrm{GII} 4 \mathrm{a}$ & I & l & $100 \%$ \\
\hline & GII5 & - & - & - & GII5.I & 2 & 2 & $100 \%$ \\
\hline & - & GII5a & - & - & - & 1 & 1 & $100 \%$ \\
\hline & - & - & - & - & GII5b & I & I & $100 \%$ \\
\hline & - & - & - & GII5c & - & I & I & $100 \%$ \\
\hline & - & - & - & - & GII5d & I & 1 & $100 \%$ \\
\hline & - & GII5e & GII5e & GII5e & GII5e & 4 & i & $25 \%$ \\
\hline & GII6 & GII6.I & GII6.I & - & GII 6.2 & 4 & 3 & $75 \%$ \\
\hline & - & - & - & Gll6a & - & I & I & $100 \%$ \\
\hline & GII6b & GII6b.I & GII6b.I & GII6b & GII6b.I & 5 & 2 & $40 \%$ \\
\hline Total Gls & 17 & 16 & 16 & 17 & 21 & & & \\
\hline Unique Gls & 12 & 10 & 9 & 13 & 17 & & & \\
\hline \% Unique & $71 \%$ & $63 \%$ & $56 \%$ & $76 \%$ & $81 \%$ & & & \\
\hline
\end{tabular}

Note: Rows represent 37 different genomic locations where Gls were found in at least one strain. These positions are listed according to the relative location in strain K96243. Different GI names indicate different gene content [see Additional file 3]. Gls shared among strains at the same relative genomic location are indicated (bold). $* \mathrm{Gls} \mathrm{I}-16$ in strain $\mathrm{K} 96243$ were previously described [II], whereas GII6b is reported here for the first time.

Third, we identified new, distinct GIs that were present at genomic locations other than the 16 previously described from K96243 [11]. These new GIs were identified by searching for integrations of large DNA blocks in the ACT alignment of the five strains; these integrations were found in both single strains and multiple strains. If these occurred in multiple strains at the same genomic location, the gene contents of the inserts were compared to determine if they represented distinct GIs. This process identi- fied GIs present at 21 additional genomic locations (Table 2).

Fourth, the contents of all identified GIs were analyzed to determine if they met the criteria described above. Most identified GIs contained low $\% \mathrm{G}+\mathrm{C}$, sequence composition different from the core backbone composition, and were large inserts. In addition, insertions usually were located adjacent to tRNA genes, flanking direct repeats or 
Table 3: GIs located next to tRNA genes in five B. pseudomallei strains.

\begin{tabular}{|c|c|c|c|c|c|c|c|}
\hline & \multirow[t]{2}{*}{ Gls } & \multicolumn{5}{|c|}{ Gls located next to tRNA genes } & \multirow[t]{2}{*}{ tRNA-SSR } \\
\hline & & K96243 & $1710 b$ & $1106 a$ & MSHR668 & MSHR305 & \\
\hline \multirow[t]{35}{*}{ Chromosome I } & Glla & & & & $x$ & & \\
\hline & $\mathrm{Gl} 2$ & $x$ & & & & & tRNA-Phe \\
\hline & G14 & $x$ & & & & & tRNA-Met \\
\hline & GI4.I & & $x$ & & & & tRNA-Met \\
\hline & GI4.2 & & & $x$ & & & tRNA-Met \\
\hline & Gl4.3 & & & & $x$ & & \\
\hline & GI4.4 & & & & & $x$ & tRNA-Met \\
\hline & GI5 & $x$ & & & & & tRNA-Leu \\
\hline & Gl5a & & $x$ & & & & \\
\hline & GI5a.I & & & $x$ & $x$ & & \\
\hline & Gl5a.2 & & & & & $x$ & \\
\hline & Gl6b & & $x$ & & & & tRNA-Pro \\
\hline & $\mathrm{Gl7}$ & $x$ & & & & & \\
\hline & GI7.I & & $x$ & & & & tRNA-Arg \\
\hline & GI7.2 & & & $x$ & & & \\
\hline & GI7.3 & & & & $x$ & & tRNA-Arg \\
\hline & GI7.4 & & & & & $x$ & \\
\hline & GI8d & & & & & $x$ & tRNA-Cys \\
\hline & G19 & $x$ & & & & & tRNA-Ser \\
\hline & $\mathrm{G} 19 \mathrm{a}$ & & & & $x$ & & \\
\hline & Gl9b & & & & $x$ & & tRNA-Gly \\
\hline & GI9c & & & & $x$ & & tRNA-Pro \\
\hline & G19c.I & & & & & $x$ & tRNA-Pro \\
\hline & GIIO & $x$ & & & $x$ & & tRNA-Thr \\
\hline & GII0.I & & $x$ & & & & \\
\hline & GII0.2 & & & $x$ & & & tRNA-Thr \\
\hline & GII0.3 & & & & & $x$ & tRNA-Thr \\
\hline & GIII & $x$ & & & & & tRNA-Ala \\
\hline & GIII.I & & & $x$ & & & \\
\hline & GIII.2 & & & & & $x$ & tRNA-Ala \\
\hline & GII2 & $x$ & & & & & tRNA-Arg \\
\hline & GII2.I & & $x$ & & & & \\
\hline & GII 2.2 & & & $x$ & & & \\
\hline & GII 2.3 & & & & $x$ & & tRNA-Arg \\
\hline & GII 2.4 & & & & & $x$ & \\
\hline \multirow[t]{6}{*}{ Chromosome 2} & GII3 & $x$ & & & & & tRNA-Ser \\
\hline & GII3.I & & $x$ & & & & \\
\hline & GII3.2 & & & $x$ & & & \\
\hline & GII3.3 & & & & $x$ & & \\
\hline & GII3.4 & & & & & $x$ & \\
\hline & GII5d & & & & & $x$ & \\
\hline
\end{tabular}

Note: The specific tRNA genes that facilitate tRNA-site specific recombination (tRNA-SSR) at some of these genomic locations are listed. In many cases tRNA-SSR (bold) has facilitated the insertion of multiple, distinct Gls in different strains at the same genomic location.

insertion sequence (IS) elements, or mobility genes such as integrases or transposases.

GIs appear to be a major source of genomic diversity within $B$. pseudomallei as each strain has a distinct set of GIs (Table 2). We identified 71 distinct GIs among the five strains that we examined. The number of GIs identified from each strain varied: a total of $17,16,16,17$, and 21 GIs were identified from strains K96243, 1710b, 1106a, MSHR668, and MSHR305, respectively. For all five strains, more than half of the GIs found in that strain were unique to that particular strain. At seven of the 37 genomic locations, GIs were present in all five strains. However, none of these seven genomic locations contained the same GI across all five strains. These data illustrate that particular GIs are present in some B. pseudomallei strains but absent in other strains. We note that we identified a new GI in strain K96243, GI16b, which is located between GI16 and GI13 on chromosome 2 in K96243. Locations of GIs in strains 1710b, 1106a, MSHR668 and 
MSHR305 aligned against the relative locations of the original 16 GIs from K96243 are illustrated in Figure 1 and listed in Table 2. Sizes of the GIs ranged from $3.91 \mathrm{~Kb}$ for GI7.4 in MSHR305 to $107.94 \mathrm{~Kb}$ for GI6b in $1710 \mathrm{~b}$. Interestingly, dinucleotide signatures [17] 'GC' and 'CG' have the highest frequencies in all GIs regardless of the actual \%G+C [see Additional file 2]. This and other information for all GIs is presented in Additional file 3.

We identified GIs in B. pseudomallei based upon the aligned differences observed across multiple genomes, which could be explained by horizontal acquisition of DNA segments from other organisms. Recently Vernikos and Parkhill [18] presented an interesting machine learning approach for identifying GIs in bacteria based upon weights of eight criteria, including: the interpolated variable order motif (IVOM) score, presence/absence of integrase, presence/absence of phage-related protein domains, size, RNA, density, repeats, and insertion points. Their studies in three different bacterial genera, Salmonella, Staphylococcus, and Streptococcus, suggest that GIs can be seen as a superfamily of mobile elements, with core and variable structural features, rather than a well-defined family.

ii) Nomenclature of B. pseudomallei genomic islands

Each of the five strains of B. pseudomallei that we examined contained unique GIs. Thus, it seems quite likely that the number of GIs discovered in this species will continue to increase as additional genome sequences become available. To facilitate scientific communication, we feel it is necessary to create a nomenclature for these important sources of genomic diversity. We propose a GI nomenclature system based upon differences in terms of locations and gene content between the newly identified GIs and the original 16 GIs identified in K96243. K96243 was cho-

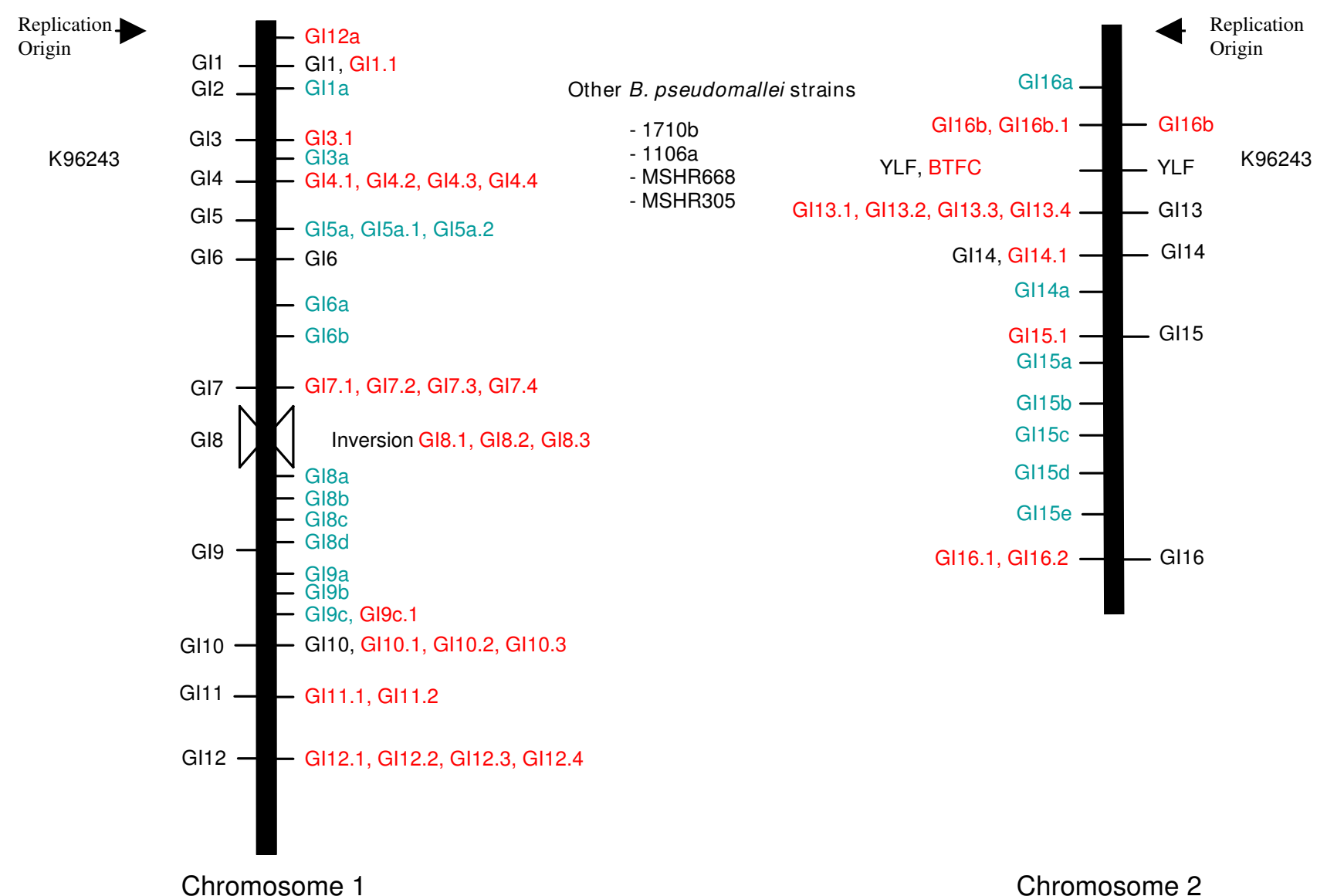

Figure I

Genomic locations of 7 I GIs on chromosomes I and 2 in B. pseudomallei. Gls identified from strains I7I0b, I I06a, MSHR668, and MSHR305 (inside of lines) are compared to the original I6 Gls identified from strain K96243 [I I] (outside of lines). The genomic location of two mutually exclusive genomic regions, BTFC (B. thailandensis-like flagella and chemotaxis gene cluster) and YLF (Yersinia-like fimbrial gene cluster), [12] is also indicated. 
sen as the reference genome for GI locations because it was the first $B$. pseudomallei strain whole-genome sequenced and it is commonly used in many other studies. Detailed criteria for the proposed nomenclature are as follows:

\section{a) Precedence}

Any GIs found at the same relative GI locations and containing the same gene contents will be given the same name as the GIs previously identified in K96243. For example, strains $1710 \mathrm{~b}$ and $1106 \mathrm{a}$ contain GIs at same position as GI1 of K96243 and both the GIs in these strains have similar gene composition to GI1 of K96243. Hence, these GIs also are called GI1.

\section{b) Unique gene composition}

Any GIs containing different gene content but located at the same reference genomic location will be given the same name as GIs in K96243 but with suffix differentiation based upon the order of their discovery (e.g., x.1, x.2, x.3). For example, strain MSHR668 contains a GI at the same location as GI1 in K96243 but it has different gene content. Thus, the GI in MSHR668 is designated as GI1.1, which indicates that this GI is located at the same location as GI1 but has different gene content. Some GIs may be found at the same genomic location and share highly similar sets of genes but contain differing numbers of mobility genes, suggesting different recombination events or mechanisms. These would be considered distinct GIs and given unique suffix designations. This criterion describes the difference between GI14.1 of MSHR668 and GI14 in K96243, 1710b, 1106a, and MSHR305. Both GIs contain similar genes but GI14.1 contains an extra pair of IS407A transposase genes, indicating that the GIs resulted from different recombination events. Using this criterion, we are able to identify different GIs that are located at the same reference location across multiple strains of $B$. pseudomallei.

\section{c) Unique genomic location}

Any novel GIs discovered at genomic locations located between two consecutive reference GI locations in K96243 will be named with a lower case letter (a to z) suffix indicating a new insertion position. Whenever possible these will be named in alphabetic ascending order consistent with the numerical order (e.g., GI9: GI9a, GI9b, GI9c: GI10). However, subsequent GI discovery studies may require a non-sequential naming, which we think is still preferable to a more complex system with additional digital codes. In this study, MSHR668 contains a new GI located between the genomic locations of GI1 and GI2 in K96243; this new GI is named GI1a. We use "a" because this is the first new GI discovered in this genomic region. We also used this rule to name GI9a, GI9b and GI9c, which are new GIs located in between the K96243 genomic positions of GI9 and G10. It is important to note that these new reference positions sometimes contain multiple GIs. In such a case, the GI name will be consistent with naming rule $b$ and a "dot numeral" is added as a suffix (e.g., ".1"). In this study, we identified a new GI, GI9c, in MSHR668 but strain MSHR305 also contains a new GI at this same genomic location but with different gene content. The MSHR305 GI is therefore named GI9c.1 in accordance with naming rules $\mathrm{b}$ and $\mathrm{c}$.

Using these three naming conventions, we are able to name new GIs discovered from multiple B. pseudomallei genomes according to their genomic location order, and this system should accommodate new GIs that will be discovered as new genome sequences become available.

\section{Genetic recombination of genomic islands}

Many genomic islands in B. pseudomallei are created by site-specific recombination mechanisms. Site-specific recombination (SSR) involves the alignment of identical, or nearly identical sequences followed by the breaking and joining (crossover) of the strands, resulting in the exchange of genetic material. In the case of genomic islands, these recombination events are part of the integration process of foreign genetic materials. We observed at least two SSR-types, tRNA-SSR and gene specific-SSR, among the five genomes of $B$. pseudomallei examined in this study. These two types are differentiated by the recognition sequence targets used to insert the foreign genetic material.

\section{i) $t R N A-S S R$}

GIs of $B$. pseudomallei are most commonly located next to tRNA genes. There are 9, 7, 7, 10, and 10 GIs located adjacent to tRNA genes in K96243, 1710b, 1106a, MSHR668, and MSHR305, respectively (40-60\% of all GIs found in these strains; see Additional file 1: Figures $S 1 \mathrm{a}-\mathrm{S} 1 \mathrm{j}$, and Table 3). In B. pseudomallei genomes, there are between 59 to 61 tRNA genes distributed across both chromosomes. Recombination at tRNA loci is initiated at the $3^{\prime}$ end of tRNA genes. This process creates a short, direct repeat sequence of the tRNA gene downstream of the integration site. We termed this type of recombination "tRNA-mediated site-specific recombination" or "tRNA-SSR". The genomic recognition sites are as short as a 14 bp repeat sequence (tRNA-Ser; GI13) and as long as a 56 bp repeat sequence (tRNA-Leu; GI5). Table 3 summarizes the locations of all 3 'end repeats of tRNA genes that are associated with GIs in the five B. pseudomallei genomes. We noted that not all GIs located next to tRNA genes contain the short direct repeat sequences (Table 3 ). This suggests that multiple recombination events at the same recognition site may have caused the repeat sequences to disappear. Details of tRNA sequences and their repeat sequences are presented in Additional file 4: Tables S2.1-S2.5. Numbers 
of tRNA-SSR are varied among the five B. pseudomallei strains. At least 8, 3, 2, 5, and 5 tRNA-SSR events are present in K96243, 1710b, 1106a, MSHR668, and MSHR305, respectively. In addition, recombination events at tRNA-Met, Pro, Thr, Ala, and Arg are common across the genomes (Table 3 ), suggesting that these sites serve as "genomic hotspots" for GI insertion in B. pseudomallei genomes.

To evaluate whether this type of genetic recombination is present in other Burkholderia species, we performed similar analysis on four B. mallei genomes, strains ATCC23344

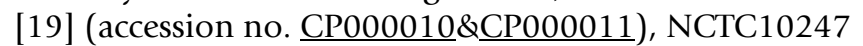
(accession no. CP000547\&CP000548), NCTC10229 (accession no. CP000545\&CP000546), and SAVP1 (accession no. CP000525\&CP000526); as well as the genome of $B$. thailandensis strain E264 (accession no. $\underline{\mathrm{CP} 000085}$ \& $\underline{\mathrm{CP} 000086})$. Similar to B. pseudomallei, tRNASSR events at tRNA-Pro genes were found in all four $B$. mallei strains. In addition, these integration events were all mediated by a well-known insertion element, IS407A [19] (Figure 2). In B. thailandensis E264, Yu and colleagues [20] previously identified 15 different genomic islands, known as Bt-GI1 to Bt-GI15. We aligned all 15 GIs from this $B$. thailandensis strain to the relative genomic locations of GIs in B. pseudomallei. In B. thailandensis E264, we found one tRNA-SSR event present at tRNA-Ser and two at tRNA-Arg, corresponding to the same genomic locations as GI13, GI7.1, and GI12 in B. pseudomallei, respectively [see Additional file 5]. In addition, the 3 'end repeat downstream of tRNA-Arg in B. pseudomallei K96243 (GI12) and $B$. thailandensis E264 has caused a recombinant tRNA-Ser. This is an unusual event in B. pseudomallei genomes and it is not known if this extra tRNA-Ser is functional in these $B$. pseudomallei and B. thailandensis strains. Figure 3 illustrates the recombination events that we propose caused these new recombinant tRNAs-Ser in B. pseudomallei
K96243 and B. thailandensis E264. Details of all tRNA-SSR events and 3 'end tRNA repeat sequences in the $B$. thailandensis and $B$. mallei genomes are presented in Additional file 4: Tables S2.6 - S2.10.

\section{ii) Gene specific recombination}

We identified a site-specific recombination event at the mutS gene (DNA mismatch repair protein) in strain MSHR305 that is associated with GI8c of B. pseudomallei. The insertion of GI8c caused two direct repeats of $15 \mathrm{bp}$ located on genes BURPS305_7225 (DNA mismatch repair protein) and BURPS305_7284 (mutS, DNA mismatch repair protein). The genes have different sizes and are only partially identical. A 15 bp recognition site, 5'ACGCCGATGATGCAG-3', of the BURPS305_7225 gene is predicted to be involved in this genetic recombination event (Figure 4). Additionally, we found that recombination at the mutS gene was also associated with Bt-GI4 in $B$. thailandensis E264. To our knowledge, this mutS-specific recombination is reported here for the first time.

Recognition sites at the 3'end of various tRNA genes and mutS genes in B. pseudomallei and B. thailandensis serve as hotspots for genetic recombination in these bacterial species. tRNA-SSR has been reported in other Gram-negative bacteria such as the VPI-2 pathogenicity island of Vibrio cholerae, which is associated with the sequence of tRNASer [21]. Recombination at a specific gene also has been reported at the glr (glutamate racemase) gene of Helicobacter pylori where it is served as the recognition or integration site of the cag pathogenicity islands [22].

\section{Gene contents and predicted functional roles of Gls}

Overall, most GIs contain genes known to be involved with genomic mobility and genetic recombination. These genes include transposase, integrase, conjugal plasmid protein, recombinase, invertase, and resolvase genes.

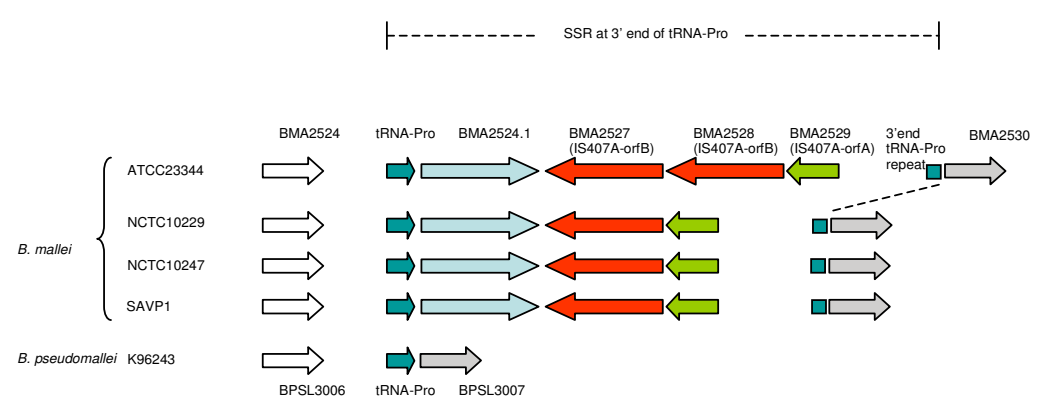

\section{Figure 2}

Site-specific recombination (SSR) at tRNA-Pro in B. mallei causes a 3' end repeat 26 bp downstream of the recombination site. This site contains insertion element IS407A, which is common in $B$. mallei genomes. This suggested that IS407A was present in other mobile genetic elements when they were originally introduced to $B$. mallei genomes and the recombination event was associated with tRNA-Pro. 


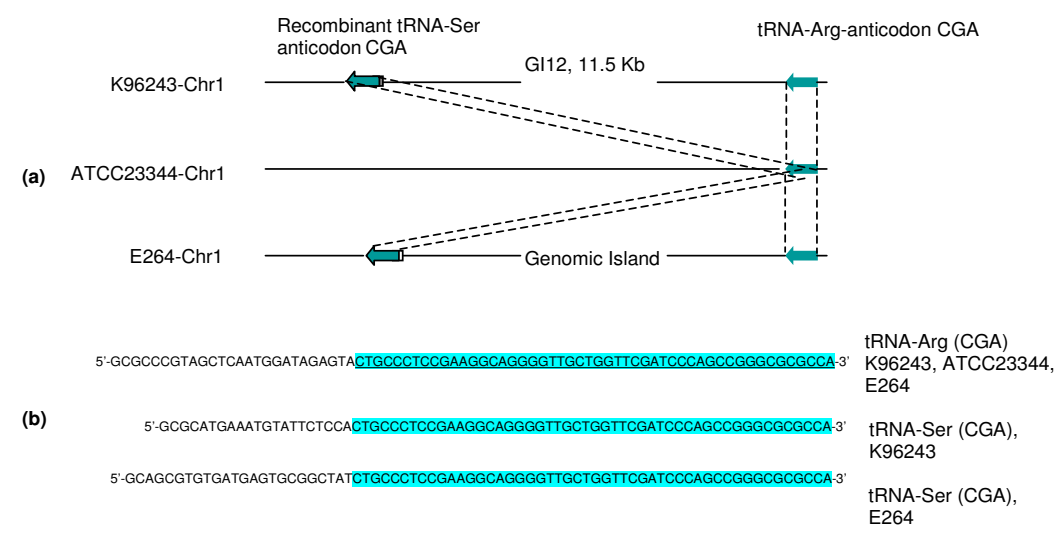

\section{Figure 3}

Predicted site-specific recombination (SSR) events at tRNA-Arg (CGA) in B. pseudomallei K96243 and B. thailandensis E264 and the relative genomic region in B. mallei ATCC23344. (a) An extra recombinant tRNA-Ser (CGA) was created in the genomes of these two bacterial strains by SSR; parallel dash lines indicate identical regions of the tRNA genes. (b) Nucleotide sequences of the tRNA-Arg and the recombinant tRNA-Ser; the predicted SSR-recognition site of tRNA-Arg in strains K96243 and E264 is underlined, and the identical regions between these two tRNA genes are highlighted in blue.

More than $80 \%$ of GIs contain at least one transposase genes. Transposase genes are known as major components of insertion elements in bacterial genomes. Most common IS elements located in the genomic islands are members of the IS3 family, including IS407A and ISB 1 [23]. IS407A was found in most GIs from all strains except MSHR305, whereas ISBp1 was only found in GIs of strains $1710 \mathrm{~b}$ and 1106a. MSHR305 contains unique IS elements in its GIs, such as ISPsy16, ISAfe1, ISAfe4, and ISrso12, that are uncommon in GIs from other strains. This suggests that GIs in B. pseudomallei originated from different sources, as they were brought to the species by different insertion elements.

To predict functional roles of GIs in B. pseudomallei, we classified all GIs from the five B. pseudomallei strains into four different functional categories: prophage, metabo- lism, pathogenicity, and unknown. GIs can contain genes that are predicted to be involved in more than one of these functions. In this study, we classified GIs based upon the functional role of a majority of the genes. Abundance of genomic islands for these four major categories in five strains of B. pseudomallei is shown in Figure 5. Details of all 4 categories are as follows:

\section{i) Prophages}

Many GIs in B. pseudomallei are prophages or prophagelike structures. The first functional prophage in B. pseudomallei was identified in K96243 [11]. Following UV induction, strain K96243 produced at least one lysogenic phage known as $\Phi$ K96243, which was able to infect $B$. mallei. DNA sequence analysis of ФK96243 indicates that it is associated with GI2, which contains approximately $36.3 \mathrm{~Kb}$ of genes necessary for bacteriophage biogenesis.

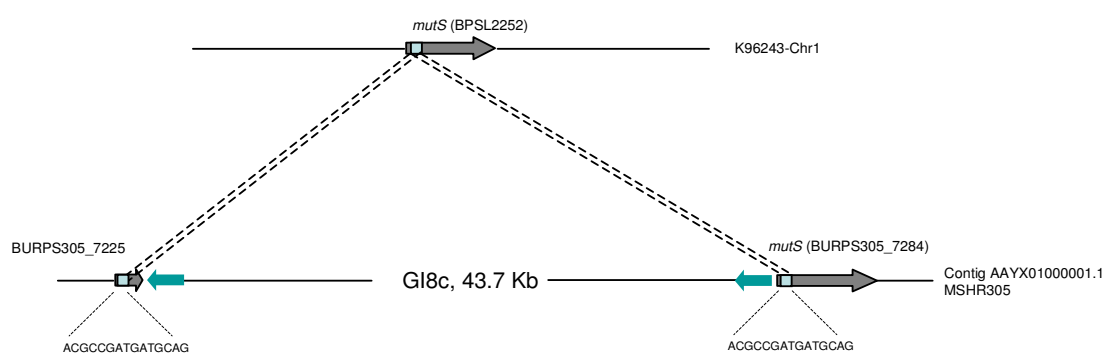

\section{Figure 4}

Site-specific recombination at the mutS gene of B. pseudomallei MSHR305. This event caused a short direct repeat I 5 bp downstream of GI8c. GI8c is a putative prophage of MSHR305. 


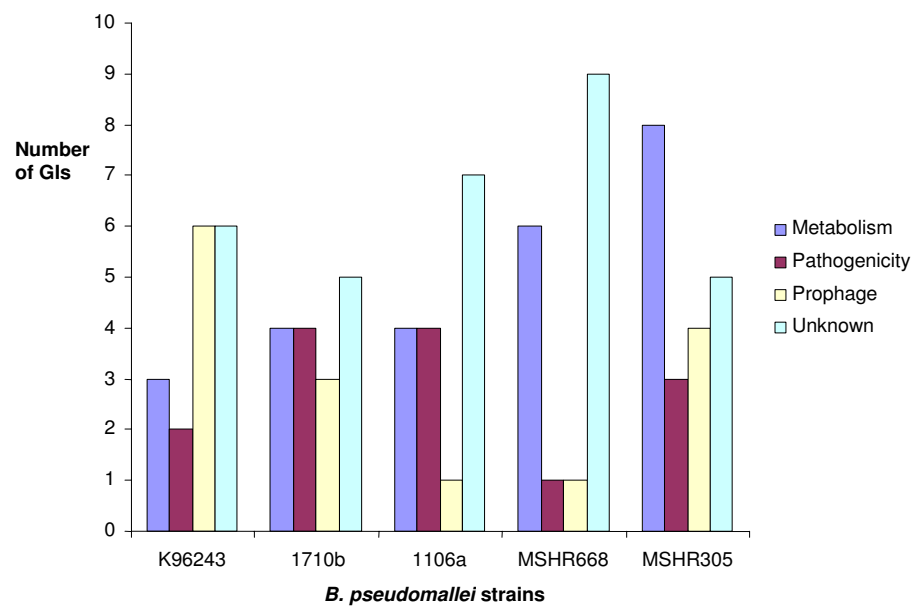

Figure 5

Prevalence of Gls with four predicted functions in five reference B. pseudomallei strains. Interestingly, strains MSHR668 and MSHR305 from Australia contain a high number of Gls associated with metabolism.

We note that GI2 is located directly downstream of tRNAPhe and contains a direct repeat ( $45 \mathrm{bp}$ ) of the 3 ' end sequence of tRNA-Phe. This repeat sequence is believed to be generated during bacteriophage lysogenization at the recognition site, known as attB, which is the $3^{\prime}$ end sequence of tRNA-Phe. GI2 is not present in the other four $B$. pseudomallei genomes examined here, but it is found in other B. pseudomallei strains such as Pasteur 52237 (accession no.AAHV00000000) and S13 (accession no. AAHW00000000) (data not shown).

Another well characterized prophage in Burkholderia species is associated with site-specific recombination at the 3 'end of tRNA-Pro in B. thailandensis E125. ФE125, a temperate bacteriophage from $B$. thailandensis, has been shown to insert as a lysogen in B. mallei ATCC23344 by site-specific recombination at the 3'end of tRNA-Pro [24]. This type of prophage in $B$. pseudomallei was firstly described in strain 1026b [25].

Bacteriophage induction occurred spontaneously during normal growth of strain $1026 \mathrm{~b}$ in liquid culture. We found that strain $1710 \mathrm{~b}$ contains a prophage (GI6b) that is similar to the functional prophage of $1026 \mathrm{~b}$, but it is two-fold larger in size. Both prophages are similar since they are associated with tRNA-SSR at tRNA-Pro. Surprisingly, GI6b contains two 3'end tRNA-Pro repeats (49 and 23 bp; Additional file 4: Table S2.2), suggesting multiple site-specific recombination events. There are no data demonstrating that GI6b in strain $1710 \mathrm{~b}$ is a functional prophage or can be induced. However, this putative prophage does contain genes for bacteriophage biogenesis and also a putative phospholipase gene
(BURPS1710b_1675), suggesting that specialized transduction has occurred. Several studies have shown that phospholipase genes are potential virulence genes in $B$. pseudomallei [26,27].

Site-specific recombination that forms bacterial prophages is not only associated with specific tRNAgenes, but also with specific gene targets. MSHR305 contains a putative prophage in GI8c that is associated with the mutS gene. Recombination has created a small $15 \mathrm{bp}$ repeat downstream of the genomic island, as described earlier. We also note that strain 1106 a contains only one prophage (GI10.2) in its genome, which is associated with site-specific recombination at tRNA-Thr. Again, there are no data confirming that the GI10.2 is a functional prophage, although its structure does contain bacteriophage biogenesis genes.

\section{ii) Metabolism}

GIs containing metabolic genes are variable across different B. pseudomallei strains and populations, which may affect bacterial fitness and be related to specific environmental niches. Nineteen of the $71(26.7 \%)$ GIs we identified in $B$. pseudomallei contain metabolic genes [see Additional file 3]. GI14 and GI14.1 are similar in term of gene contents and both contain genes with predicted metabolic functions. Hence, both represent potential metabolic islands. The genes in these islands include peptidase enzymes such as collagenase (BPSS0666), alpha-ketoglutarate-dependent taurine dioxygenase (BPSS0665), and x-prolyl-dipeptidyl aminopeptidase (BPSS0654). These genes are predicted to be involved in amino acid metabolism but there are no studies on their actual functional roles. We used a PCR 
assay to query the presence/absence of the BPSS0654 taurine dioxygenase gene across large $B$. pseudomallei strain collections from Thailand and Australia. We found that this gene is present in $99 \%$ and $97 \%$ of tested strains from these two countries, respectively [see Additional file 6]. We note that strains MSHR668 and MSHR305 contain six and eight GIs with metabolic genes, respectively (Figure 5). Metabolic GI gene BURPS305_5421 (bacterial extracellular solute-binding protein) in MSHR305 has a very limited distribution, occurring in less than $10 \%$ of tested strains from Thailand and $56 \%$ of tested strains from Australia [see Additional file 6].

\section{iii) Pathogenicity}

We have identified two different groups of genes in $B$. pseudomallei GIs that could have functional roles in bacterial pathogenicity, especially bacterial adherence. They are genes that encode for filamentous hemagglutinin proteins (FHA) and two-partner secretion systems (TPS).

FHA genes in B. pseudomallei are located on different genomic islands and varied among strains. Strain 1106a contains three different clusters of fha genes located at GI5a.1, GI11.1, and GI16.1; strain 1710b contains two clusters (GI5a and GI16.1); strain K96243 contains one cluster at GI16; and strains MSHR668 and MSHR305 contain only one cluster at GI5a.1 and GI5a.2, respectively. Homologs of fha genes have been reported in other Gramnegative bacteria where they have functional roles in virulence. When we compared the fhaB gene, which is the largest gene in each fha gene cluster, to its homolog in Bordetella pertussis, we found high similarity to all three $B$. pseudomallei gene clusters. Thus, it seems likely that all three genes encode a filamentous hemagglutinin domain protein in B. pseudomallei [see Additional file 7]. More recently, Sim and Yu et al. reported that the experimental mutation one fhaB gene (BPSS2053, equivalent to fhaB3 gene in our study) in B. pseudomallei DD503 caused the reduction of microbial adherence to human epithelial cells [28]. We also examined the occurrence of this gene among diverse $B$. pseudomallei populations and found that fhaB copy number varied among the strains. Most B. pseudomallei strains from Thailand and Australia contained either two clusters of fhaB genes (cluster I from GI5a/ GI5a.1/GI5a.2 and cluster III from GI16/GI16.1), or a fhaB gene from cluster III (GI16/GI16.1) alone. In contrast, cluster II found in strain 1106a (GI11.1) was very rare. Details of the distribution of fhaB gene clusters in $B$. pseudomallei are demonstrated in Figure $6 \mathrm{a}$ and a list of tested strains is provided in Additional file 6 .

Two-partner secretion systems in B. pseudomallei were first described by Brown and colleagues in 2002 [29]. They reported that this particular two-partner secretion system was encoded by genes $b p a A B$, which were located in the GIs of strain 08 but absent in many other $B$. pseudomallei strains used in their study. We have determined that these genes are located in GI15d of MSHR305 and not observed in the other four B. pseudomallei genome sequences examined here. We examined the frequency of the bpaA gene (the largest gene in GI15d) among diverse B. pseudomallei strains and found that it was rare. Only $2.3 \%$ of strains contained this gene and, interestingly, these strains were only found in clinical isolates from Australia [see Figure 6b; Additional file 6]. B. pseudomallei virulence determinants are not well understood, but animal models have demonstrated that different strains do differ in their virulence levels [30,31] and this could be related to variation in GIs and GI content.

\section{iv) Unknown functional roles}

The functional roles of many genomic islands in B. pseudomallei (32\%) is unknown; most genes found in B. pseudomallei GIs have only hypothetical protein predictions that do not match any characterized proteins databases.

The four categories of functional roles of GIs described above are based primarily upon computer predictions and only a minimal number of laboratory studies. Direct functional studies of GI genes are very rare, and most functional genomics studies rely upon $B$. pseudomallei gene conservation compared to other species. Almost all comparative genomic studies among $B$. pseudomallei strains identify differences primarily among genomic islands genes [25,32,33]. In a 2006 study by Duangsonk and colleagues [32], GI genes identified by suppression subtractive hybridization (SSH) between two different $B$. pseudomallei strains were used to develop a variable amplicon typing (VAT) scheme. VAT was applied successfully to population genetics problems and differentiated groups of $B$. pseudomallei strains that caused different clinical outcomes. Most recently, genes of five GIs from K96243, including GI2, GI6, GI9, GI11, and GI16, were used to measure frequency values among clinical and environmental B. pseudomallei strains from Thailand [34]. There were no significant differences between environmental and disease-associated isolates for these genes. However, because only GIs from K96243 were used, this study was not representative of the large GI diversity observed among multiple B. pseudomallei genomes. Still, this is one of the first examples attempting to correlate these very important genomic components to clinically relevant phenotypes. Because of the importance of GIs in the accessory genome of $B$. pseudomallei, they may play important roles in various phenotypic differences within the $B$. pseudomallei species. Studies of GI genes in various fields of research, such as population genetics and functional genomics, warrant further investigation but this must be based upon pan-genomic analysis using all available data.

\section{Conclusion}

The data presented here suggest that a large number of GIs have been acquired by horizontal acquisitions and that 


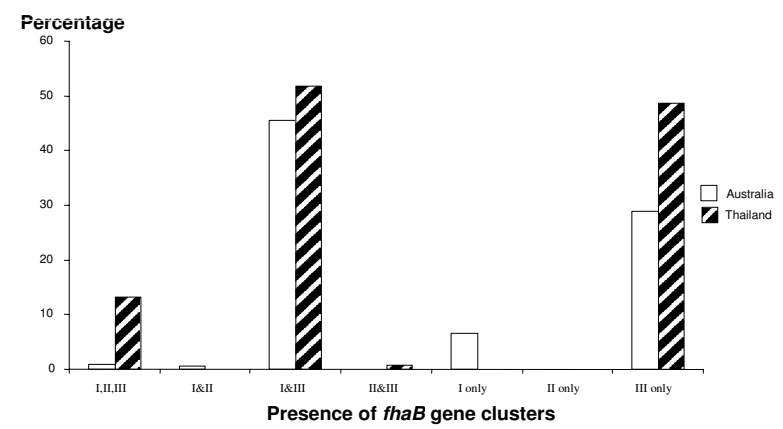

(a)

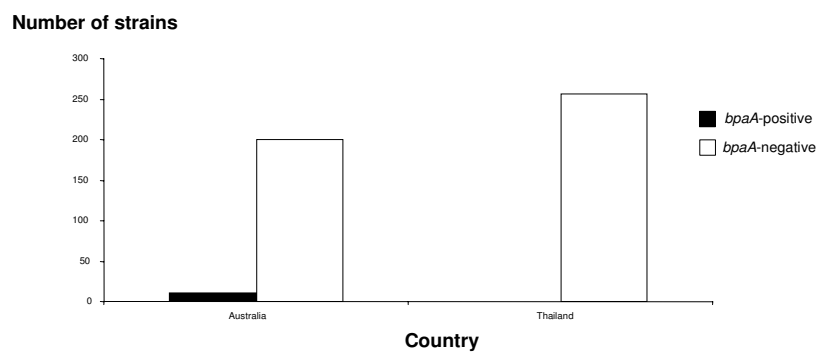

(b)

\section{Figure 6}

Distribution (presence) of GI genes within diverse B. pseudomallei populations. (a) Presence of fhaB gene clusters. (b) Presence of $b p a A$ gene. We note that most $B$. pseudomallei strains contained either two clusters of the fhaB gene, clusters I and III, or cluster III alone. In addition, the $b p a A$ gene was found only in clinical isolates of $B$. pseudomallei from Australia.

these GIs represent a major source of genomic diversity in $B$. pseudomallei. The proposed nomenclature suggested above will be important for effective communication in the research community and for cataloging the highly variable GIs. tRNA-mediated site-specific recombination appears to be an important mechanism for horizontal gene transfers of GIs. The differential presence of GIs in multiple strains also demonstrates the limited phylogenetic distribution of mobile genetic elements in this bacterial species. GIs are part of the accessory genome, which have not been studied thoroughly in B. pseudomallei. Various fields of postgenomics, such as population genetics and functional genomics of GIs, are worthy of further investigations.

\section{Methods}

\section{Genomic Data}

Genomes of five clinical B. pseudomallei strains were used in this study. These strains included K96243, 1710b, 1106a, MSHR668, and MSHR305. Clinical information and genomic features of all five strains are summarized in Table 1.

\section{Comparative genomics, genome alignment, and bioinformatics of genomic island}

Comparisons of the genome sequences for most aspects of this study were performed using ACT (Artemis comparison tool) [15]. Each genomic comparison file was generated from NCBI- Local BLAST program, which is available in BioEdit Sequence Alignment Editor [35]. Dinucleotide signatures among all genomic islands were analyzed using an in-house Java program. Dinucleotide frequencies and indices were calculated according to Karlin and Burge [17]. Circular diagrams of all genomes used in this study were made by using CGView [36].

\section{Experimental examination of genomic islands}

SYBR-Green real-time PCR assays were developed to examine the presence/absence of genomic island genes across multiple strains of $B$. pseudomallei. Specific genes from six genomic islands were selected and used as the targets for PCR. These included three different clusters of fha $B$ genes located in several different GIs; $b p a A$, a known gene encoding for a two-partner secretion system; and two metabolic genes, BPSS0654 of GI14 and BURPS305_5421 of GI14a. Details of PCR primers and target genes are described in Additional file 8. We used genomic DNA samples from five genome-sequenced strains, K96243, $1710 \mathrm{~b}, 1106 \mathrm{a}, \mathrm{MSHR} 668$ and MSHR305 as positive and/ or negative PCR controls. A total of 468 genomic DNA samples were used in the analysis.

\section{Authors' contributions}

AT conceived of the study, performed major analyses, and drafted the manuscript. BRL performed validation of most experiments. RKA, SMBS, and JMBS provided bioinformatic assistances and analyses. MM and VW provided DNA samples for testing throughout the study. TSB pro- 
vided shotgun genome sequences of MSHR305. WCN, SJP, BJC, DMW and PK participated in the design and coordination of the study and manuscript preparation. All authors have read and approved the manuscript.

\section{Additional material}

\section{Additional file 1}

Circular diagrams. Figure S1a. Circular diagram of the artificial chromosome 1 of MSHR305. Figure S1b. Circular diagram of the artificial chromosome 2 of MSHR305. Figure S1c. Circular diagram of genomic islands on chromosome 1 of K96243. Figure S1d. Circular diagram of genomic islands on chromosome 2 of K96243. Figure S1e. Circular diagram of genomic islands on chromosome 1 of $1710 b$. Figure S1f. Circular diagram of genomic islands on chromosome 2 of $1710 \mathrm{~b}$. Figure S1g. Circular diagram of genomic islands on chromosome 1 of 1106 . Figure S1h. Circular diagram of genomic islands on chromosome 2 of 1106a. Figure S1i. Circular diagram of genomic islands on chromosome 1 of MSHR668. Figure S1j. Circular diagram of genomic islands on chromosome 2 of MSHR668

Click here for file

[http://www.biomedcentral.com/content/supplementary/1471-

2164-9-566-S1.doc]

\section{Additional file 2}

Dinucleotide frequency. Figure S2. Analysis of dinucleotide frequencies of all genomic islands compared to the conserved regions in $\mathrm{B}$. pseudomallei genomes. Frequencies of dinucletides "CG and $G C$ " are relatively high regardless of the total $\% \mathrm{G}+\mathrm{C}$ of the GIs.

Click here for file

[http://www.biomedcentral.com/content/supplementary/14712164-9-566-S2.doc]

\section{Additional file 3}

CDS coordinates of genomic islands. Table S1. Details of all genomic islands described in this study.

Click here for file

[http://www.biomedcentral.com/content/supplementary/14712164-9-566-S3.xls]

\section{Additional file 4}

tRNA-SSR. Table S2.1 tRNA-SSR distribution in B. pseudomallei K96243. Table S2.2 tRNA-SSR distribution in B. pseudomallei $1710 b$. Table S2.3 tRNA-SSR distribution in B. pseudomallei 1106a. Table S2.4 tRNA-SSR distribution in B. pseudomallei MSHR668. Table S2.5 tRNA-SSR distribution in B. pseudomallei MSHR305. Table S2.6 tRNA-SSR distribution in B. thailandensis E246. Table S2.7 tRNA-SSR distribution in B. mallei ATCC23344. Table S2.8 tRNA-SSR distribution in B. mallei NCTC10247. Table S2.9 tRNA-SSR distribution in B. mallei NCTC10229. Table S2.10 tRNA-SSR distribution in B. mallei SAVP1.

Click here for file

[http://www.biomedcentral.com/content/supplementary/1471-

2164-9-566-S4.xls]

\section{Additional file 5}

Relative genomic island locations in B. pseudomallei and B. thailandensis. Table S3. Relative genomic island locations in 5 B. pseudomallei strains and B. thailandensis E264.

Click here for file

[http://www.biomedcentral.com/content/supplementary/14712164-9-566-S5.xls]

\section{Additional file 6}

Distribution of GIs. Table S4. Distribution of selected genomic island genes in B. pseudomallei strains.

Click here for file

[http://www.biomedcentral.com/content/supplementary/14712164-9-566-S6.xls]

\section{Additional file 7}

Amino acid sequences of fhaB genes. Figure S3. Showing homology of amino acid sequences of $3 \mathrm{fhaB}$ genes in $\mathrm{B}$. pseudomallei and a reference amino acid sequence (IRWR_chainA) for filamentous hemagglutinin of Bordetella pertussis. This suggests that three fhaB genes of $\mathrm{B}$. pseudomallei may have the same functional role as the filamentous hemagglutinin, a virulence effector molecule in $\mathrm{B}$. pertussis. Click here for file [http://www.biomedcentral.com/content/supplementary/14712164-9-566-S7.doc]

\section{Additional file 8}

PCR primers. Table S5. PCR primers used in this study. Click here for file [http://www.biomedcentral.com/content/supplementary/14712164-9-566-S8.doc]

\section{Acknowledgements}

AT holds a Career Development Award (May 2007-April 2009) from the NIH Pacific Southwest Regional Center of Excellence. This work was funded by NIH-NIAID grants U54 AI-065359 and U0IAI-075568.

\section{References}

I. White NJ: Melioidosis. Lancet 2003, 36 I(9370): 17/5-1722.

2. Maharian B, Chantratita N, Vesaratchavest M, Cheng A, Wuthiekanun V, Chierakul W, Chaowagul W, Day NPJ, Peacock SJ: Recurrent melioidosis in patients in northeast Thailand is frequently due to reinfection rather than relapse. Journal of Clinical Microbiology 2005, 43(I 2):6032-6034.

3. Wuthiekanun V, Peacock SJ: Management of melioidosis. Expert Review of Anti-infective Therapy 2006, 4(3):445-455.

4. Suputtamongkol $Y$, Chaowagul W, Chetchotisakd $P$, Lertpatanasuwun N, Intaranongpai S, Ruchutrakool T, Budhsarawong D, Mootsikapun P, Wuthiekanun V, Teerawatasook N, et al.: Risk factors for melioidosis and bacteremic melioidosis. Clinical Infectious Diseases 1999, 29(2):408-413.

5. Suputtamongkol Y, Hall AJ, Dance DA, Chaowagul W, Rajchanuvong A, Smith MD, White NJ: The epidemiology of melioidosis in Ubon Ratchatani, northeast Thailand. International Journal of Epidemiology 1994, 23(5): 1082-1090.

6. Howe C, Sampath A, Spotnitz M: The pseudomallei group: a review. Journal of Infectious Diseases 197I, 124:598-606.

7. Rotz LD, Khan AS, Lillibridge SR, Ostroff SM, Hughes JM: Public health assessment of potential biological terrorism agents. Emerging Infectious Diseases 2002, 8(2):225-230.

8. Elliott JH, Anstey NM, Jacups SP, Fisher DA, Currie BJ: Communityacquired pneumonia in northern Australia: low mortality in a tropical region using locally-developed treatment guidelines. International Journal of Infectious Diseases 2005, 9(I):15-20.

9. Wiersinga WJ, Poll T van der, White NJ, Day NP, Peacock SJ: Melioidosis: insights into the pathogenicity of Burkholderia pseudomallei. Nature Reviews Microbiology 2006, 4(4):272.

10. Cheng AC, Currie BJ: Melioidosis: epidemiology, pathophysiology, and management. Clinical Microbiology Reviews 2005, I8(2):383-416.

II. Holden MTG, Titball RW, Peacock SJ, Cerdeno-Tarraga AM, Atkins T, Crossman LC, Pitt T, Churcher C, Mungall K, Bentley SD, et al:: Genomic plasticity of the causative agent of melioidosis, Burkholderia pseudomallei. PNAS 2004, I0I(39): I 4240-I 4245. 
12. Tuanyok A, Auerbach RK, Brettin TS, Bruce DC, Munk AC, Detter JC, Pearson T, Hornstra H, Sermswan RW, Wuthiekanun V, et al.: A horizontal gene transfer event defines two distinct groups within Burkholderia pseudomallei that have dissimilar geographic distributions. Journal of Bacteriology 2007, I 89(24):9044-9049.

13. Kim HS, Schell MA, Yu Y, Ulrich RL, Sarria SH, Nierman WC, DeShazer $D$ : Bacterial genome adaptation to niches: divergence of the potential virulence genes in three Burkholderia species of different survival strategies. BMC Genomics 2005, 6: 174.

14. Hacker J, Carniel E: Ecological fitness, genomic islands and bacterial pathogenicity: A Darwinian view of the evolution of microbes. EMBO Reports 200I, 2(5):376-38I.

I5. Carver TJ, Rutherford KM, Berriman M, Rajandream M-A, Barrell BG, Parkhill J: ACT: the Artemis comparison tool. Bioinformatics 2005, 2 I ( I 6):3422-3423.

16. Hacker J, Kaper JB: Pathogenicity islands and evolution of microbes. Annual Review of Microbiology 2000, 54(I):64I-679.

17. Kariin S, Burge C: Dinucleotide relative abundance extremes: a genomic signature. Trends in Genetics 1995, I I (7):283-290.

18. Vernikos GS, Parkhill J: Resolving the structural features of genomic islands: A machine learning approach. Genome Research 2008, I 8(2):331-342.

19. Nierman WC, DeShazer D, Kim HS, Tettelin H, Nelson KE, Feldblyum T, Ulrich RL, Ronning CM, Brinkac LM, Daugherty SC, et al. Structural flexibility in the Burkholderia mallei genome. PNAS 2004, I 0 I(39): |4246-1425I.

20. Yu Y, Kim HS, Chua H, Lin C, Sim S, Lin D, Derr A, Engels R, DeShazer $D$, Birren $B$, et al.: Genomic patterns of pathogen evolution revealed by comparison of Burkholderia pseudomallei, the causative agent of melioidosis, to avirulent Burkholderia thailandensis. BMC Microbiology 2006, 6(I):46.

21. Murphy RA, Boyd EF: Three pathogenicity islands of Vibrio cholerae can excise from the chromosome and form circular intermediates. Journal of Bacteriology 2008, I90(2):636-647.

22. Censini S, Lange C, Xiang Z, Crabtree JE, Ghiara P, Borodovsky M, Rappuoli R, Covacci A: cag, a pathogenicity island of Helicobacter pylori, encodes type I-specific and disease-associated virulencefactors. PNAS |996, 93(25): |4648-| 4653.

23. Woo P, Leung $P$, Tsoi H-W, Chan B, Que T-L, Yuen K-Y: Characterization of a novel insertion sequence, ISBpI, in Burkholderia pseudomallei. Archives of Microbiology 2002, I 77(3):267-273.

24. Woods DE, Jeddeloh JA, Fritz DL, DeShazer D: Burkholderia thailandensis EI 25 harbors a temperate bacteriophage specific for Burkholderia mallei. Journal of Bacteriology 2002 I 84( I 4):4003-40 I 7

25. DeShazer D: Genomic diversity of Burkholderia pseudomallei clinical isolates: subtractive hybridization reveals a Burkholderia mallei-specific prophage in B. pseudomallei I 026b. Journal of Bacteriology 2004, I 86( I 2):3938-3950.

26. Korbsrisate S, Suwanasai N, Leelaporn A, Ezaki T, Kawamura Y, Sarasombath S: Cloning and characterization of a nonhemolytic phospholipase $\mathbf{C}$ gene from Burkholderia pseudomallei. Journal of Clinical Microbiology 1999, 37( I I ):3742-3745.

27. Tuanyok A, Tom M, Dunbar J, Woods DE: Genome-wide expression analysis of Burkholderia pseudomallei infection in a hamster model of acute melioidosis. Infection and Immunity 2006, 74(I 0):5465-5476.

28. Sim SH, $\mathrm{Yu} Y$, Lin $\mathrm{CH}$, Karuturi RKM, Wuthiekanun $\mathrm{V}$, Tuanyok $\mathrm{A}$ Chua $\mathrm{HH}$, Ong C, Paramalingam SS, Tan G, et al.: The core and accessory genomes of Burkholderia pseudomallei: implications for human melioidosis. PLoS Pathogens 2008 4(10): 1000178.

29. Brown NF, Logue CA, Boddey JA, Scott R, Hirst RG, Beacham IR: Identification of a novel two-partner secretion system from Burkholderia pseudomallei. Molecular Genetics and Genomics 2004, 272(2):204-2I5

30. Barnes JL, Ketheesan N: Route of infection in melioidosis. Emerging Infectious Diseases 2005, I I (4):638-639.

31. Ulett GC, Currie BJ, Clair TW, Mayo M, Ketheesan N, Labrooy J, Gal D, Norton R, Smith CA, Barnes J, et al.: Burkholderia pseudomaIlei virulence: definition, stability and association with clonality. Microbes and Infection 200I, 3(8):62I-63I.

32. Duangsonk K, Gal D, Mayo M, Hart CA, Currie BJ, Winstanley C: Use of a variable amplicon typing scheme reveals considerable variation in the accessory genomes of isolates of Burkhol- deria pseudomallei. Journal of Clinical Microbiology 2006, 44(4): I 323-1334.

33. Ou K, Ong C, Koh SY, Rodrigues F, Sim SH, Wong D, Ooi CH, Ng $\mathrm{KC}$, Jikuya $\mathrm{H}$, Yau CC, et al.: Integrative genomic, transcriptional, and proteomic diversity in natural isolates of the human pathogen Burkholderia pseudomallei. Journal of Bacteriology 2005, I 87( I 2):4276-4285.

34. Tumapa S, Holden M, Vesaratchavest M, Wuthiekanun V, Limmathurotsakul D, Chierakul W, Feil E, Currie B, Day N, Nierman W, et al.: Burkholderia pseudomallei genome plasticity associated with genomic island variation. BMC Genomics 2008, 9(I): 190.

35. Hall T: BioEdit: a user-friendly biological sequence alignment editor and analysis program for Windows 95/98/NT. Nucleic Acids Symposium Series 1998, 41:95-98.

36. Stothard $P$, Wishart DS: Circular genome visualization and exploration using CGView. Bioinformatics 2005, 2 I (4):537-539.
Publish with Biomed Central and every scientist can read your work free of charge

"BioMed Central will be the most significant development for disseminating the results of biomedical research in our lifetime. "

Sir Paul Nurse, Cancer Research UK

Your research papers will be:

- available free of charge to the entire biomedical community

- peer reviewed and published immediately upon acceptance

- cited in PubMed and archived on PubMed Central

- yours - you keep the copyright
BioMedcentral 